\title{
Posterior Titanium Screw Fixation without Debridement of Infected Tissue for the Treatment of Thoracolumbar Spontaneous Pyogenic Spondylodiscitis
}

\author{
Mauro Dobran, Maurizio Iacoangeli, Davide Nasi, Niccolo Nocchi, \\ Alessandro Di Rienzo, Lucia di Somma, Roberto Colasanti, \\ Carmela Vaira, Roberta Benigni, Valentina Liverotti, Massimo Scerrati \\ Department of Neurosurgery, Umberto I General Hospital, Università Politecnica delle Marche, Ancona, Italy
}

\begin{abstract}
Study Design: Retrospective study.
Purpose: The aim of our study was to analyze the safety and effectiveness of posterior pedicle screw fixation for treatment of pyogenic spondylodiscitis (PSD) without formal debridement of the infected tissue.

Overview of Literature: Posterior titanium screw fixation without formal debridement of the infected tissue and anterior column reconstruction for the treatment of PSD is still controversial.

Methods: From March 2008 to June 2013, 18 patients with PSD underwent posterior titanium fixation with or without decompression, according to their neurological deficit. Postero-lateral fusion with allograft transplantation alone or bone graft with both the allogenic bone and the autologous bone was also performed. The outcome was assessed using the visual analogue scale (VAS) for pain and the Frankel grading system for neurological status. Normalization both of C-reactive protein (CRP) and erythrocyte sedimentation rate was adopted as criterion for discontinuation of antibiotic therapy and infection healing. Segmental instability and fusion were also analyzed.

Results: At the mean follow-up time of 30.16 months (range, 24-53 months), resolution of spinal infection was achieved in all patients. The mean CRP before surgery was $14.32 \pm 7.9 \mathrm{mg} / \mathrm{dL}$, and at the final follow-up, the mean CRP decreased to $0.5 \pm 0.33 \mathrm{mg} /$ $\mathrm{dL}(p<0.005)$. Follow-up computed tomography scan at 12 months after surgery revealed solid fusion in all patients. The VAS before surgery was $9.16 \pm 1.29$ and at the final follow-up, it improved to $1.38 \pm 2.03$, which was statistically significant $(p<0.05)$. Eleven patients out of eighteen (61.11\%) with initial neurological impairment had an average improvement of 1.27 grades at the final follow-up documented with the Frankel grading system.

Conclusions: Posterior screw fixation with titanium instrumentation was safe and effective in terms of stability and restoration of neurological impairment. Fixation also rapidly reduced back pain.
\end{abstract}

Keywords: Pyogenic; Infection; Discitis; Posterior surgery; Titanium pedicle screws

\footnotetext{
Received Sep 14, 2015; Revised Oct 4, 2015; Accepted Oct 4, 2015

Corresponding author: Mauro Dobran

Clinica di Neurochirurgia, Università Politecnica delle Marche, Ospedali Riuniti di Ancona,

Via Conca \#71, Ancona 60020, Italy

Tel.: +39 0715964567, Fax: +39 0715964575, E-mail: dobran@libero.it
} 


\section{Introduction}

The larger population of older and immunocompromised people in recent decades has contributed to an increasing number of spinal infections [1]. Pyogenic spondylodiscitis (PSD) is the most common, and the term "spondylodiscitis" refers to the infection of the intervertebral disc by a pathogen and secondarily to the osteomyelitis of the adjacent end plates, usually occurring in conjunction each other [2]. Due to the low specificity of signs and symptoms, the detection of PSD occurs late and, when there is radicular or spinal cord compression, the infection has already reached an advanced stage $[3,4]$. The treatment of PSD is essentially conservative with bed rest, external spinal immobilization, and administration of antibiotics [1-7]. However, surgery is indicated in front of a neurologic deficit, failure of antibiotic therapy and presence of spine instability [1-10]. The main goals of surgical treatment of PSD are early decompression of the spinal cord in presence of neurological deficits, infected tissue debridement and stabilization of the involved vertebral segment. Placement of metallic instrumentation in the setting of a spinal infection is still controversial and remains a taboo for most spinal surgeons, especially without aggressive debridement of infected tissue [10].

The primary reason against the use of titanium or other metallic implants is that any prosthetic implant may be a substrate for bacterial adherence and biofilm formation that in turn reduce antibiotic penetration. However, the use of posterior instrumentation in spinal infection may still be justified, because the implant transverses healthy tissue and minimally contacts the lap of infection, and provides stability in inflammation control (similar to use in osteomyelitis cases as in long bones). To date, few reports have discussed treating PSD with the posterior approach only $[1,5,11,12]$.

The purpose of this study was to evaluate the safety and efficacy of titanium pedicle screw implants for posterior fixation without removal of infected tissue and anterior support for PSD of the thoracic and lumbar spine.

\section{Materials and Methods}

From March 2008 through June 2013, 18 patients (13 men and 5 women) with PSD of the thoracic or lumbar spine underwent posterior screw fixation with titanium instrumentation associated with posterolateral fusion.
Postern-lateral fusion was performed with allograft transplantation alone or bone graft with both the allogenic and autologous bone. Autologous bone graft was used only in patients undergoing laminectomy and was harvested from the spinous processes and laminae.

The indications for surgical intervention were a neurological deficit, instability of the spine, or obtaining tissue for diagnosis and failure of a conservative treatment. Posterior decompression was performed in presence of a neurological deficit. Laminectomy only was done for posterior decompression, based on the level of infection, without debridement of the infected disc or endplates (Fig. 1). However, culture specimen was obtained in all patients from a limited biopsy of the disc space through a posterior approach (laminectomy or partial hemilaminectomy in patients without neurological deficit). In case of pre-existing severe kyphotic deformity or complete lack of anterior support brought about by the infection, the use of only posterior approach was not indicated and we used an anterior or anterolateral approach with the aim to restore anterior column followed by a posterior fixation. These cases were not included in this series.

The mean age at the time of surgery was 62.05 years (range, 20-84 years), and the mean follow-up time was 30.16 months (range, 24-53 months). All the patients had reported severe back pain. Among them, 11 had neurological impairment. All patients except two (88\%) suffered various associated comorbidities (Table 1 ). The affected levels were the thoracic spine in 8 patients, thoracolumbar junction in one, lumbar spine in 8 , and lumbosacral junction in one patient. Antibiotics were administrated until the erythrocyte sedimentation rate (ESR) and C-reactive protein (CRP) levels returned normal.

Serial computed tomography (CT) scan, magnetic resonance imaging and anteroposterior and lateral radiographs were performed immediately after surgery and at 3 , 6, and 12 months after operation, and then yearly (Fig. 2). Neurological deficits were grouped according to Frankel classification. Postoperative care included brace usage for 3 to 6 months, based on follow-up imaging and laboratory test. Back pain was evaluated with the visual analogue scale (VAS, 0-10). Statistical analysis was performed using SPSS ver. 20 (SPSS Inc., Chicago, IL, USA). The univariate analysis of data was carried out by the Pearson chi-square test for discrete variables, and the $t$-test for the continuous ones. 

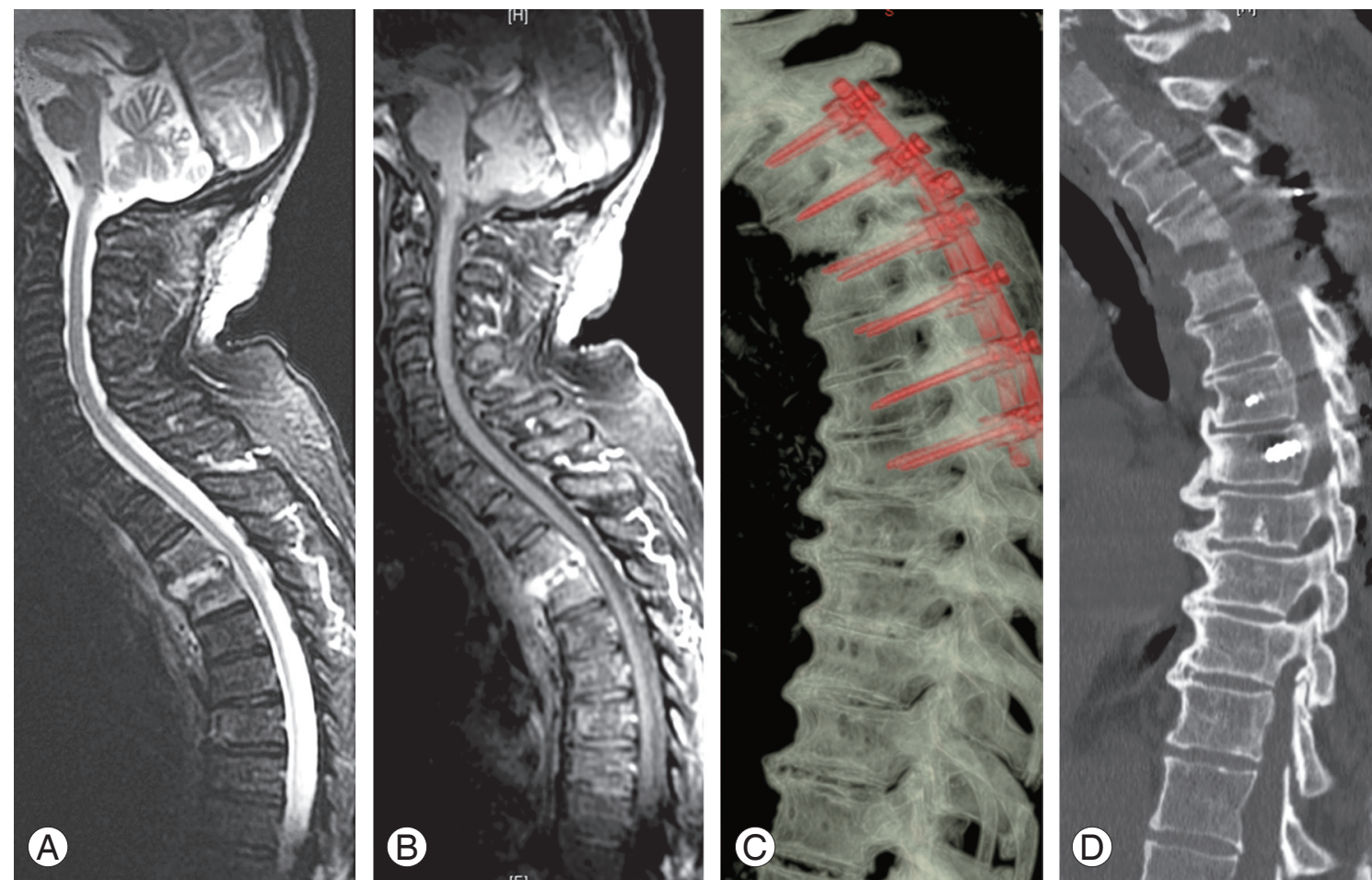

Fig. 1. Case number 17 of Table 2. (A, B) Preoperative magnetic resonance imaging images showed a pyogenic spondylodiscitis at T3-T4 with bony destruction and segmental instability associated with an epidural abscess causing initial spinal cord compression. (C, D) Postoperative computed tomography scan with sagittal and 3D reconstructions showed posterior long fixation (with pedicle screws and rods) and decompression at T3-T4.

Table 1. Comorbidities of patients with pyogenic spondylodiscitis

\begin{tabular}{lc} 
Comorbidities & No. of patients \\
\hline Diabetes mellitus & 6 \\
\hline End stage of renal disease & 4 \\
Intravenous drug user & 2 \\
\hline Rheumatoid arthritis & 1 \\
\hline Ulcerative colitis & 1 \\
\hline Prostate cancer & 1 \\
\hline Chronic hepatitis & 1 \\
No comorbidity & 2 \\
\hline Total & 18 \\
\hline
\end{tabular}

\section{Results}

At the mean follow-up time of 30.16 months (range, 2453 months), resolution of spinal infection was achieved in all patients (defined with the normalization of inflammatory markers, changes in imaging reads, and resolution of pain). The mean duration of intravenous antibiotic therapy was 2.11 months (range, 1-11 months), while the mean
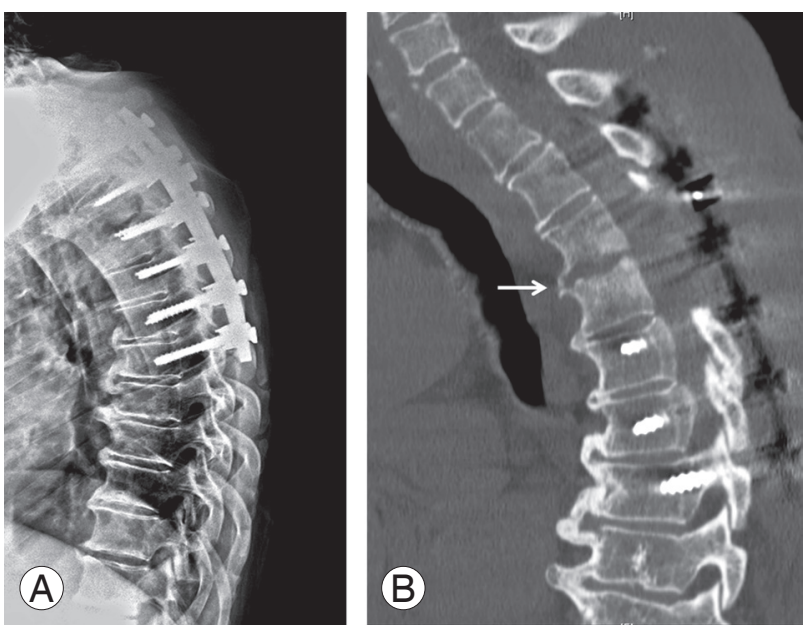

Fig. 2. (A) At the six months follow-up, lateral radiograph revealed no segmental kyphosis or hardware breakage. (B) At the 1 year followup, a computed tomography scan with sagittal reconstruction demonstrated signs of bony fusion between T3 and T4 (white arrow).

duration of oral antibiotic therapy was 5.33 months (range, 1-12 months). Solid fusion at CT scan was achieved at the 12-months follow-up in all patients. There was no relapse of infection at the lesion level in any of the patients during 


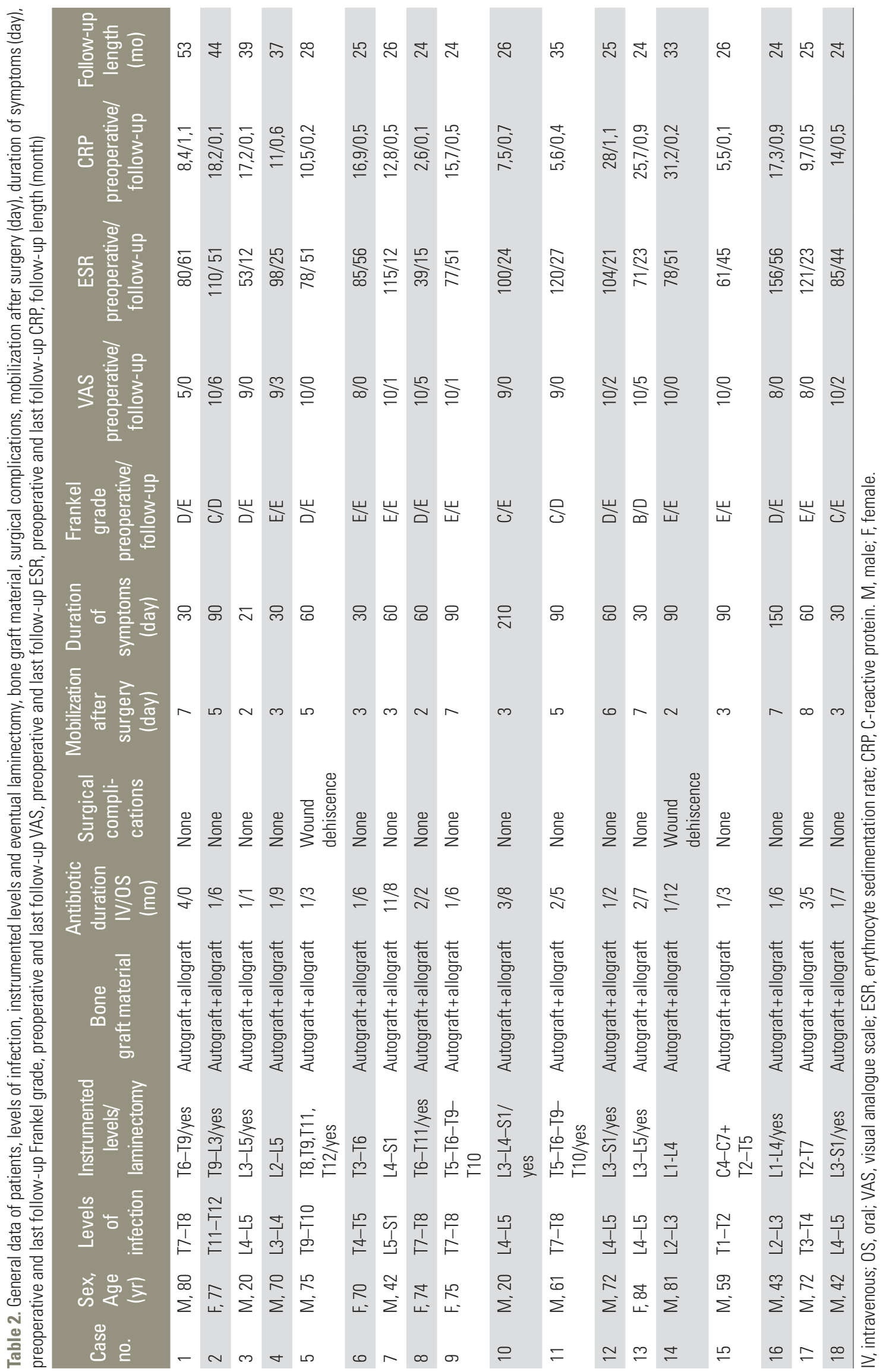


Table 3. Results of intraoperative culture

\begin{tabular}{lc} 
Pathogen & No. of patients \\
\hline Staphylococcus aureus & 8 \\
\hline Methicillin-sensitive & 5 \\
\hline Methicillin-resistant & 3 \\
\hline Escherichia coli & 3 \\
\hline Streptococcus agalactiae & 1 \\
Streptococcus pyogenes & 1 \\
Culture negative & 5 \\
\hline Total & 18 \\
\hline
\end{tabular}

Table 4. Results of the primary site of infection

\begin{tabular}{lc} 
Primary site of infection & No. of patients \\
Urinary tract & 5 \\
Bacteraemia due to endocarditic disease & 4 \\
Infection at a vascular access site & 2 \\
\hline Pneumonia & 1 \\
\hline Intestinal infection & 1 \\
Sepsis after prostatic biopsy & 1 \\
Unknown & 4 \\
\hline Total & 18 \\
\hline
\end{tabular}

follow-up and no titanium implants were removed. The average VAS of back pain before surgery was $9.16 \pm 1.29$ at the final follow-up improved to $1.38 \pm 2.03$, which was statistically significant $(p<0.05)$. Eleven patients with neurological impairment (Frankel grading system value $1-4$ ) at the final follow-up improved 1.27 grades. On admission, the mean erythrocyte sedimentation rate was $90.61 \mathrm{~mm} /$ $\mathrm{hr}$ (range, 39-156 mm/hr) and CRP level was $14.32 \pm 7.9$ $\mathrm{mg} / \mathrm{dL}$ (range, 2.6-25.7 mg/dL). The erythrocyte sedimentation rate and CRP level became normal within 3 months after surgery for all patients and at the final follow-up the mean CRP decreased to $0.5 \pm 0.33 \mathrm{mg} / \mathrm{dL}(p<0.005)$. General data of patients, levels of infection, instrumented levels and eventual laminectomy, bone graft material, surgical complications, mobilization after surgery, duration of symptoms, preoperative and last follow-up Frankel grade, preoperative and last follow-up VAS, preoperative and last follow-up ESR, preoperative and last follow-up CRP, and follow-up times are summarized in Table 2.

Thirteen patients out of eighteen (61.11\%) had an intraoperative positive culture and Staphylococcus aureus was the most common pathogen detected (Table 3). The culture-negative patients with evidence of pyogenic inflammation were submitted to histological examination of tissue. The primary site of infection was documented in 14 patients out of $18(77.77 \%)$ and results are shown in Table 4.

\section{Discussion}

Spinal infection accounts for $2 \%-7 \%$ of all cases of musculoskeletal infections [13]. Although the mortality rate has been reduced in developed countries, ranging from 2 and 4\%, PSD remains a challenging pathology to diagnose and treat [14]. In the present study, 18 patients with PSD of the thoracic or lumbar spine underwent posterior screw fixation with titanium instrumentation associated with posterolateral fusion. Until now, the role of spinal instrumentation in the presence of infection has been hardly discussed. In recent years direct spinal fixation following the debridement of the lesion of tuberculous spondylitis and pyogenic discitis has been conducted; this was following the knowledge in osteomyelitis of providing stability to long bones using metal implants being effective in inflammation control even when metallic or cement implants were placed in an infected area [5]. Several retrospective studies revealed better results in patients with spondylodiscitis operated with posterior instrumentation than in those not fixed [2,14-18]. Lin et al. [1] reported that long posterior instrumentation with short posterior or posterolateral fusion in the treatment of PSD was effective in terms of infection control, kyphotic correction, and maintenance of correction and restoration of neurological impairment, and most of the patients achieved a good functional outcome after surgery. More recently, Mohamed et al. [5] showed that long posterior pedicle screws fixation combined with aggressive antibiotic therapy resulted in resolution of spinal infection in 15 patients. In these two series, no relapse of infection was observed after surgery with titanium implants. Also, Lee et al. [10] demonstrated that stabilizing the spine with pedicle screws and titanium mesh prevented the deformity in patients with tuberculous spondylitis and the metallic implants did not prohibit infection control.

In our series of patients operated with titanium screws and rods, the resolution of spinal infection was achieved in all patients (defined with the normalization of inflammatory markers and imaging), and resolution of pain and improvement of the VAS scores were seen (from an aver- 
age of 9.16 preoperatively to 1.38 after surgery; $p<0.05$ ). Eleven patients out of 18 with neurological deficit had an improvement of 1.27 grades according to the Frankel system. The erythrocyte sedimentation rate and CRP levels became normal for all patients within 3 months after surgery and antibiotics treatment. Finally, solid fusion was identified at 12 months follow-up in all 18 patients.

For use of titanium and other metal hardware, there is the belief that all foreign materials (including metallic implants and allografts) tend to act as substrates for bacterial adherence and biofilm formation thus reducing antibiotic penetration [19]. Such biofilm formation has been known to result in persistence of the infection by blocking antibiotic penetration into the infected areas. In vitro studies suggest that biofilm can block antibiotics, phagocytes and other humoral immune responders reaching target bacteria, making such infections relatively resistant to host defensive mechanisms and antibiotics [19-21]. There are, however, a number of variables in terms of the characteristics and composition of the metal in question [19]. Titanium alloy (the metal used in this series) is less prone to colonization than stainless steel [15-21]. Furthermore, in our patients, the use of posterior instrumentation in spinal infection may still be justified as the implant traverses relatively healthy tissue, whereas the anterior cage is seated into the lap of infection.

In case of an epidural abscess, some authors advocated the complete removal of infected material to avoid not only mechanical compression but also septic thrombi. In this series, we treated only patients with a purulent fluid collection [1]. For this reason, the posterior approach with limited laminectomies and passage of soft silicon catheters for extraction or irrigation of pus allowed in all patients the complete evacuation of infected material. In the event that significant phlegmon or granulation tissue is encountered, surgical removal of infected tissue is usually required for an anterior approach. In such cases, posterior fixation and decompression alone are not indicated.

In all patients, we performed postero-lateral fusion with allograft transplantation alone or bone graft with both the allogenic and autologous bone. There have been few reports about the use of allograft in the treatment of PSD. Among these, An et al. [12] reported the use of allograft alone or bone graft with both the allogenic and autologous bone for posterior lumbar interbody fusion after debridement of the intervertebral disc space. Moreover, previous studies have reported that the allogenic bone was used instead of the autologous bone in the treatment of osteomyelitis that occurred in the long bone. Also, in our experience long posterior fixation with postero-lateral fusion lesion can provide stability and prevent kyphotic deformity. However, we don't only use posterior approach in case of pre-existence severe kyphotic deformity or complete lack of anterior support brought about by the infection.

Our results suggest that posterior stabilization with titanium screws and rods in spondylodiscitis patients may be successful in addition to the antibiotic therapy. In addition regular bone fusion was achieved in all patients without the need of removing implants. Posterior stabilization of the infected spine by titanium instrumentation with fusion can facilitate nursing care, allow early patient mobilization, and thereby reducing the risk of the complications from a long-term bed rest. Posterior fixation may also reduce back pain rapidly.

\section{Conclusions}

Posterior screw fixation with titanium instrumentation was safe and effective in terms of infection control, stability and restoration of neurological impairment for treatment of PSD. In our series, after surgery, the patients presented reduction of back pain with improvement of the VAS score from an average of 9.16 preoperatively to 1.38 postoperatively $(p<0.05)$. The stabilization of the column with the reduction of pain permits an early mobilization and the quality of life seems to be more favorable.

\section{Conflict of Interest}

No potential conflict of interest relevant to this article was reported.

\section{References}

1. Lin CP, Ma HL, Wang ST, Liu CL, Yu WK, Chang MC. Surgical results of long posterior fixation with short fusion in the treatment of pyogenic spondylodiscitis of the thoracic and lumbar spine: a retrospective study. Spine (Phila Pa 1976) 2012;37:E1572-9.

2. Vcelak J, Chomiak J, Toth L. Surgical treatment of lumbar spondylodiscitis: a comparison of two methods. Int Orthop 2014;38:1425-34.

3. Duarte RM, Vaccaro AR. Spinal infection: state of the 
art and management algorithm. Eur Spine J 2013;22: 2787-99.

4. Roberto T, Daniele M, Martina C, Tiziano de G, Roberto D. Treatment of thoracolumbar spinal infections through anterolateral approaches using expandable titanium mesh cage for spine reconstruction. Scientific World Journal 2012;2012:545293.

5. Mohamed AS, Yoo J, Hart R, et al. Posterior fixation without debridement for vertebral body osteomyelitis and discitis. Neurosurg Focus 2014;37:E6.

6. Devkota P, Krishnakumar R, Renjith Kumar J. Surgical management of pyogenic discitis of lumbar region. Asian Spine J 2014;8:177-82.

7. Quinones-Hinojosa A, Jun P, Jacobs R, Rosenberg WS, Weinstein PR. General principles in the medical and surgical management of spinal infections: a multidisciplinary approach. Neurosurg Focus 2004;17:E1.

8. Schomacher M, Finger T, Koeppen D, et al. Application of titanium and polyetheretherketone cages in the treatment of pyogenic spondylodiscitis. Clin Neurol Neurosurg 2014;127:65-70.

9. Shousha M, Heyde C, Boehm H. Cervical spondylodiscitis: change in clinical picture and operative management during the last two decades. A series of 50 patients and review of literature. Eur Spine J 2015;24: 571-6.

10. Lee MC, Wang MY, Fessler RG, Liauw J, Kim DH. Instrumentation in patients with spinal infection. Neurosurg Focus 2004;17:E7.

11. Lee JS, Suh KT. Posterior lumbar interbody fusion with an autogenous iliac crest bone graft in the treatment of pyogenic spondylodiscitis. J Bone Joint Surg Br 2006;88:765-70.

12. An KC, Kim JY, Kim TH, et al. Posterior lumbar interbody fusion using compressive bone graft with allograft and autograft in the pyogenic discitis. Asian Spine J 2012;6:15-21.

13. Tyrrell PN, Cassar-Pullicino VN, McCall IW. Spinal infection. Eur Radiol 1999;9:1066-77.

14. Dimar JR, Carreon LY, Glassman SD, Campbell MJ,
Hartman MJ, Johnson JR. Treatment of pyogenic vertebral osteomyelitis with anterior debridement and fusion followed by delayed posterior spinal fusion. Spine (Phila Pa 1976) 2004;29:326-32.

15. Sundararaj GD, Babu N, Amritanand R, et al. Treatment of haematogenous pyogenic vertebral osteomyelitis by single-stage anterior debridement, grafting of the defect and posterior instrumentation. J Bone Joint Surg Br 2007;89:1201-5.

16. Klockner C, Valencia R. Sagittal alignment after anterior debridement and fusion with or without additional posterior instrumentation in the treatment of pyogenic and tuberculous spondylodiscitis. Spine (Phila Pa 1976) 2003;28:1036-42.

17. Pee YH, Park JD, Choi YG, Lee SH. Anterior debridement and fusion followed by posterior pedicle screw fixation in pyogenic spondylodiscitis: autologous iliac bone strut versus cage. J Neurosurg Spine 2008;8: 405-12.

18. Fukuta S, Miyamoto K, Masuda T, et al. Two-stage (posterior and anterior) surgical treatment using posterior spinal instrumentation for pyogenic and tuberculotic spondylitis. Spine (Phila Pa 1976) 2003; 28:E302-8

19. Sundararaj GD, Amritanand R, Venkatesh K, Arockiaraj J. The use of titanium mesh cages in the reconstruction of anterior column defects in active spinal infections: can we rest the crest? Asian Spine J 2011;5: 155-61.

20. Korovessis P, Repantis T, Iliopoulos P, Hadjipavlou A. Beneficial influence of titanium mesh cage on infection healing and spinal reconstruction in hematogenous septic spondylitis: a retrospective analysis of surgical outcome of twenty-five consecutive cases and review of literature. Spine (Phila Pa 1976) 2008; 33:E759-67.

21. Christodoulou AG, Givissis P, Karataglis D, Symeonidis PD, Pournaras J. Treatment of tuberculous spondylitis with anterior stabilization and titanium cage. Clin Orthop Relat Res 2006;444:60-5. 